\title{
A NOTE ON HYPERELLIPTIC CURVES
}

\author{
GEOFFREY MESS \\ (Communicated by Frederick R. Cohen)
}

\begin{abstract}
There is no universal hyperelliptic curve over the space of $(2 g+2)$ tuples in the projective line.
\end{abstract}

\section{INTRODUCTION}

If $C$ is a Riemann surface of genus 1 or a hyperelliptic Riemann surface of genus $g \geq 2$, then $C$ admits a $g_{2}^{1}$, i.e., a map $p: C \rightarrow \mathbf{P}^{1}$ of degree 2, branched over $2 g+2$ points. The $g_{2}^{1}$ is unique up to the action of $\mathrm{PGL}_{2} \mathbf{C}$ on $\mathbf{P}^{1}$ and (if $g=1)$ translation in $C$. We may consider the space $X_{2 g+2}$ of sets of $2 g+2$ (unordered) distinct points in $\mathbf{P}^{1}$-thus $X_{2 g+2}$ is an open set in the symmetric power $\operatorname{Sym}^{2 g+2} \mathbf{P}^{1}=\mathbf{P}^{2 g+2}$-and the tautologous bundle and subbundle $\left(\mathbf{P}^{1} \times\right.$ $\left.X_{2 g+2}, R_{2 g+2}\right)$, where the fiber of $R_{2 g+2}$ over the element $\left\{p_{1}, \ldots, p_{2 g+2}\right\}$ of $X_{2 g+2}$ is the subset $\left\{p_{1}, \ldots, p_{2 g+2}\right\}$ of $\mathbf{P}^{1}$, and ask whether there is a family $p_{Y}: Y_{g} \rightarrow X_{2 g+2}$ of hyperelliptic curves of genus $g$ over $X_{2 g+2}$, admitting a $g_{2}^{1} p: Y_{g} \rightarrow \mathbf{P}^{1} \times X_{2 g+2}$ over $X_{2 g+2}$ (i.e., such that $p_{2} \circ p=p_{Y}$, where $Z_{2 g+2}=\mathbf{P}^{1} \times X_{2 g+2}$ and $p_{2}: Z_{2 g+2} \rightarrow X_{2 g+2}$ is the projection) that is branched over $R_{2 g+2}$.

Theorem 1. For $g \geq 0$, there does not exist a family $p: Y_{g} \rightarrow Z_{2 g+2}$ satisfying the conditions above.

The referee has pointed out a simple proof for $g$ odd; our proof does not distinguish the cases $g$ odd and $g$ even (cf. Remark 4).

Proof. First we point out where the obstruction to the existence of $Y_{g}$ lies. On a fiber of $Z_{2 g+2}-R_{2 g+2}$, there is a unique element $\alpha \in$ $H^{1}\left(\mathbf{P}^{1}-\left\{p_{1}, \ldots, p_{2 g+2}\right\}, \mathbf{Z} / 2\right)$ that is the Alexander dual of $\left\{p_{1}, \ldots, p_{2 g+2}\right\}$ in $\mathbf{P}^{1}=S^{2}$. On each fiber $\alpha$ defines the desired double branched covering and $\alpha$ is left invariant by the monodromy of $\pi_{1} X_{2 g+2}$ on $H^{1}\left(\mathbf{P}^{1}-\left\{p_{1}, \ldots, p_{2 g+2}\right\}, \mathbf{Z} / 2\right)$. There exists a $Z / 2$-cohomology class on $Z_{2 g+2}-R_{2 g+2}$ that restricts to an $\alpha$

Received by the editors November 20, 1990.

1991 Mathematics Subject Classification. Primary 57M12; Secondary 14H15.

Key words and phrases. Hyperelliptic curve, braid group.

This work was partially supported by the N.S.F. 
on a fiber if and only if the differential $d_{2}: H_{\text {inv }}^{1}\left(\mathbf{P}^{1}-\left\{p_{1}, \ldots, p_{2 g+2}\right\}, \mathbf{Z} / 2\right) \rightarrow$ $H^{2}\left(X_{2 g+2}, \mathbf{Z} / 2\right)$ in the $E_{2}$ term of the spectral sequence for the fibering $t$ satisfies $d_{2}(\alpha)=0$. Otherwise $d_{2}(\alpha)$ is the obstruction to the existence of $Y_{g}$.

The case $g=0$ is degenerate, because the mapping class group of a sphere is trivial. $X_{2}$ contains the space $\mathbf{R} P^{2}$ of antipodal pairs as a deformation retract. Over $\mathbf{R} P^{2}, Z_{2}-R_{2}$ deformation retracts fiberwise to a bundle of great circles, which may be identified with the unit tangent bundle $L(4,1)$ of $\mathbf{R} P^{2}$. So there is no double cover of $Z_{2}-R_{2}$ that restricts to a nontrivial covering on each fiber.

Now we assume $g \geq 1$. By definition $\pi_{1} X_{2 g+2}$ is the braid group $B\left(2 g+2, S^{2}\right)$. Adding the relator $t_{1} \cdots t_{n}^{2} \cdots t_{1}$ to the standard presentation $\left(t_{1}, \ldots, t_{n}: t_{i} t_{i+1} t_{i}=t_{i+1} t_{i} t_{i+1},\left[t_{i}, t_{j}\right](|i-j|>1)\right)$ of Artin's braid group $B\left(n+1, \mathbf{R}^{2}\right)$ gives a presentation of $B\left(n+1, S^{2}\right)$. To see this suppose $x$ is in the kernel of the natural homomorphism from $B\left(n+1, \mathbf{R}^{2}\right)$ to $B\left(n+1, S^{2}\right) . x$ can be represented by a family of unordered $(n+1)$-tuples in $\mathbf{R}^{2}$, parametrized by a loop. The null homotopy in $X_{n+1}$ can be realized by a map of a disc to $X_{n+1}$ that restricts on the boundary to the given loop. Take the disc to be transverse to the set of $(n+1)$-tuples containing $\infty$. Then there are finitely many points in the disc, where one of the points in the $(n+1)$-tuple becomes $\infty$ and the monodromy in $B\left(n+1, \mathbf{R}^{2}\right)$ of a small loop around such a point is conjugate to $\left(t_{1} \cdots t_{n}^{2} \cdots t_{1}\right)^{ \pm 1}$ in $B\left(n+1, \mathbf{R}^{2}\right)$, as $t_{1} \cdots t_{n}^{2} \cdots t_{1}$ can be represented by holding all but the first point fixed and moving the first point around a circle containing the other $n$ points in its interior.

The kernel of the homomorphism $h: B\left(2 g+2, S^{2}\right) \rightarrow \Gamma_{0}^{2 g+2}$ is the image of $\mathrm{Z} / 2=\pi_{1} \mathrm{SO}(3)$ in $\pi_{1} X_{2 g+2}$ given by the action of $\mathrm{SO}(3)$ on $S^{2}$; it is generated by $\left(t_{1} \cdots t_{2 g+1}\right)^{2 g+2}$, as the braid $\left(t_{1} \cdots t_{2 g+1}\right)$ can be represented by a rotation of the sphere through an angle $2 \pi / 2 g+2$.

Now we consider whether the homomorphism $h: B\left(2 g+2, S^{2}\right) \rightarrow \Gamma_{0}^{2 g+2}$ lifts to a homomorphism $\bar{h}: B\left(2 g+2, S^{2}\right) \rightarrow \Gamma_{g}$ such that $h=\pi \circ \bar{h}$ where $\pi: Z(i) \rightarrow \Gamma_{0}^{2 g+2}$ is the homomorphism from the centralizer $Z(i)$ of a hyperelliptic involution $i$ to the mapping class group $\Gamma_{0}^{2 g+2} . Z(i)$ is generated by Dehn twists $\bar{t}_{1}, \ldots, \bar{t}_{2 g+1}$ on curves $C_{1}, \ldots, C_{2 g+1}$ that are invariant under the hyperelliptic involution $i . \pi\left(\bar{t}_{i}\right)=\left[t_{i}\right] \in T_{0}^{2 g+2}$, the image of $t_{i} \in B\left(2 g+2, S^{2}\right)$. If $\bar{h}$ exists, then $\bar{h}\left(t_{i}\right)=\bar{t}_{i}$ or $\bar{t}_{i} \circ i$. Since the elements $t_{i}$ are conjugate in $B\left(2 g+2, S^{2}\right)$, the elements $\bar{h}\left(t_{i}\right)$ are all conjugate in $\Gamma_{2}$; so either $\bar{h}\left(t_{i}\right)=t_{i}$ for all $i$ or $\bar{h}\left(t_{i}\right)=\bar{t}_{i} \circ i$ for all $i$. In either case $\bar{h}\left(t_{1} \cdots t_{2 g+1}^{2} \cdots t_{1}\right)=\bar{t}_{1} \cdots \bar{t}_{2 g+1}^{2} \cdots \bar{t}_{1}$ as $i$ is central and the number $4 g+2$ of letters in $t_{1} \cdots t_{2 g+1}^{2} \cdots t_{1}$ is even. But $\bar{t}_{1} \cdots \bar{t}_{2 g+1}^{2} \cdots \bar{t}_{1}=i$ in $\Gamma_{2}$. To see this draw the curves $C_{1}, C_{1} \cdot \bar{t}_{1}, \ldots, C_{1} \bar{t}_{1} \cdots \bar{t}_{2 g+1}^{2} \cdots \bar{t}_{1}$ and observe that the orientation of $C_{i}$ is reversed. Alternatively, consider the curves $H_{\theta}: y^{2}=\left(x-a_{1}\right) \cdots\left(x-a_{2 g+1}\right)\left(x-R e^{i \theta}\right)$ with $R>\left|a_{1}\right|, \ldots,\left|a_{2 g+1}\right|$. Fix $x$ with $|x|<R$. After increasing $\theta$ by $2 \pi$ the sign of $y$ has changed.

Since $h$ does not lift to $\bar{h}$, there does not exist a family $Y_{g}$.

Remarks. (1) It follows from $B\left(2 g+2, S^{2}\right)=B\left(2 g+2, \mathbf{R}^{2}\right) /\left(t_{1} \cdots t_{2 g+1}^{2} \cdots t_{1}\right)$ that $H_{1}\left(B\left(2 g+2, S^{2}\right), \mathbf{Z}\right)=\mathbf{Z} /(4 g+2)$. Another way to see this is to consider 
$n$ points with homogeneous coordinates $\left(\alpha_{1}, \beta_{1}\right)$ in $\mathbf{P}^{1}$ and write the discriminant $\prod_{i<j}\left(\alpha_{i} \beta_{j}-\alpha_{j} \beta_{i}\right)^{2}=F\left(\Sigma_{0}, \ldots, \Sigma_{n}\right)$ where $\Sigma_{i}\left(\alpha_{i}, \ldots, \alpha_{n}, \beta_{1}, \ldots, \beta_{n}\right)=$ $\sigma_{i}\left(\alpha_{1} / \beta_{1}, \ldots, \alpha_{n} / \beta_{n}\right) \beta_{1} \cdots \beta_{n}$ and $\sigma_{i}$ is the $i$ th elementary symmetric function. Then each $\Sigma_{i}$ has degree $n$ so $F$ is a homogeneous polynomial of degree $2(n-1)$. Since $F$ is irreducible, the complement of the locus $F=$ 0 in $\mathbf{P}^{n}$ has first homology group $\mathbf{Z} / 2(n-1)$. Since $\Gamma_{0}^{2 g+2}$ is obtained from $B\left(2 g+2, S^{2}\right)$ by adjoining the relation $\left(t_{1} \cdots t_{2 g+1}\right)^{2 g+2}$, it follows that $H_{1}\left(\Gamma_{0}^{2 g+2}, \mathbf{Z}\right)=\mathbf{Z} /(4 g+2)$. O. Gabber showed me this argument.

(2) Now suppose $g \geq 2$. Consider the group $\widetilde{G}=\left\langle t_{1}, \ldots, t_{2 g+1}: t_{i} t_{i+1} t_{i}=\right.$ $\left.t_{i+1} t_{i} t_{i+1}(1 \leq i \leq 2 g),\left[t_{i}, t_{j}\right](|i-j|>1),\left[t_{1}, t_{1} \cdots t_{2 g+1}^{2} \cdots t_{1}\right]\right\rangle$. Then the center of $\widetilde{G}$ is $\mathbf{Z} / 2 \oplus \mathbf{Z}$, with generators

$$
A=\left(t_{1} \cdots t_{2 g+1}\right)^{2 g+2}\left(t_{1} \cdots t_{2 g+1}^{2} \cdots t_{1}\right)^{-(g+1)}, \quad I=\left(t_{1} \cdots t_{2 g+1}^{2} \cdots t_{1}\right) .
$$

The kernels of the natural maps from $\widetilde{G}$ to $B\left(2 g+2, S^{2}\right)$ and $Z(i)$ are $I$ and $\left\langle A, I^{2}\right\rangle$, respectively. So $\widetilde{G} /\left\langle I^{2}\right\rangle$ is the pullback of the two central extensions $B\left(2 g+2, S^{2}\right), Z(i)$ of $\Gamma_{0}^{2 g+2}$. Since $H_{1}\left(\widetilde{G} /\left\langle I^{2}\right\rangle, \mathbf{Z}\right)=\mathbf{Z} / 8 g+4$, we see that the two extensions $B\left(2 g+2, S^{2}\right), Z(i)$ of $\Gamma_{0}^{2 g+2}$ arise from elements of $H^{2}\left(\Gamma_{0}^{2 g+2}, \mathbf{Z} / 2\right)$ with the same image in $\operatorname{Hom}\left(H_{2}\left(\Gamma_{0}^{2 g+2}, \mathbf{Z} / 2\right), \mathbf{Z} / 2\right)$, differing by the nontrivial element of $\operatorname{Ext}\left(H_{1}\left(\Gamma_{0}^{2 g+2}, \mathbf{Z} / 2\right), \mathbf{Z} / 2\right)$.

(We assumed $g \geq 2$ because if $g=1, \Gamma_{0}^{4}=(\mathbf{Z} / 2 * \mathbf{Z} / 3) \ltimes(\mathbf{Z} / 2)^{2}$ and the central extension corresponding to $Z(i)$ is not $\Gamma_{1}=\operatorname{SL}(2, Z)$ but rather $\operatorname{SL}(2, \mathbf{Z}) \ltimes(\mathbf{Z} / 2)^{2}$.)

(3) In fact $\mathrm{H}_{2}\left(B\left(n, S^{2}\right)\right)=\mathrm{Z} / 2$. One way to see this is to use Arnold's calculation [A] of $H_{*}\left(B\left(n, \mathbf{R}^{2}\right), \mathbf{Z}\right)$ to see that $H_{2}\left(B\left(n, \mathbf{R}^{2}\right), \mathbf{Z}\right) \cong \mathbf{Z} / 2, n \geq 4$. Since $B\left(n, S^{2}\right)$ is obtained from $B\left(n, \mathbf{R}^{2}\right)$ by adding a relator $t_{1} \cdots t_{n-1}^{2} \cdots t_{1}$ that has infinite order in homology, Hopf's formula [Br] shows that $H_{2}\left(B\left(n, \mathbf{R}^{2}\right)\right)$ $\cong H_{2}\left(B\left(n, S^{2}\right)\right)$.

The 5-term exact sequence for $P B\left(n, \mathbf{R}^{2}\right) \rightarrow B\left(n, \mathbf{R}^{2}\right) \rightarrow \Sigma_{n}$ shows that the natural map $H_{2}\left(B\left(n, \mathbf{R}^{2}\right)\right) \rightarrow H_{2}\left(\Sigma_{n}, \mathbf{Z}\right)$ is onto. Since this map factors through $H_{2}\left(\Gamma_{0}^{n}, \mathbf{Z}\right)$, the exact sequence $H_{2}\left(B\left(n, S^{2}\right), \mathbf{Z}\right) \rightarrow H_{2}\left(\Gamma_{0}^{n}, \mathbf{Z}\right) \rightarrow$ $\mathbf{Z} / 2 \rightarrow 0$ from the 5-term sequence for $\mathbf{Z} / 2 \rightarrow B\left(n, S^{2}\right) \rightarrow \Gamma_{0}^{n}$ is split: $H_{2}\left(\Gamma_{0}^{n}, \mathbf{Z}\right) \cong \mathbf{Z} / 2 \oplus \mathbf{Z} / 2$. It follows that $H_{2}\left(\Gamma_{2}, \mathbf{Z}\right) \cong \mathbf{Z} / 2$, which is confirmed by $[\mathrm{C} 2, \mathrm{BCT}]$.

(4) Since $B\left(2 g+2, S^{2}\right)$ and $\Gamma_{0}^{2 g+2}$ have abelianization $Z /(4 g+2)$ while $Z(i)$ has abelianization $Z /(4 g+2)$ for $g$ even and $Z /(8 g+4)$ for $g$ odd, there can be no lift $\bar{h}$ of the homomorphism $h$ in case $g$ is odd, so for odd $g$ the theorem becomes more elementary.

Finally let us remark that if we consider the double cover $\widetilde{X}_{2 g+2}$ of $X_{2 g+2}$, with fundamental group an extension of $A_{2 g+2}$ by the pure braid group $P B\left(2 g+2, S^{2}\right)$, the obstruction vanishes because $d_{2}(\alpha)$ is the Bockstein of the nontrivial element of $H^{1}\left(B\left(2 g+2, S^{2}\right), \mathrm{Z} / 2\right)$, and the Bockstein homomorphism is natural. If $q=1$, then one can alternatively consider the cover $X_{4}^{\prime}$ of $X_{4}$ corresponding to the subgroup $S_{3}$ of the quotient $S_{4}$ defined by permuting the points; $\pi_{1} X_{4}^{\prime}=\mathrm{SL}_{2} \mathrm{Z}$.

The homomorphism $\mathrm{SL}_{2} \mathbf{Z} \rightarrow \Gamma_{0}^{4}=\mathrm{PSL}_{2} \mathbf{Z} \times(\mathbf{Z} / 2)^{2}$ lifts to $\mathrm{SL}_{2} \mathbf{Z} \times(\mathbf{Z} / 2)^{2}$. Since $X_{2 g+2}$ has the homotopy type of a $\mathrm{SL}_{2} \mathrm{C}$ bundle over a 
$K\left(B\left(2 g+2, S^{2}\right), 1\right)$, there can be no obstruction to constructing the required family of genus 1 curves. As a check, the family can be exhibited

$$
Z^{2}=\frac{(Q X-P Y)\left(A X^{3}+B X^{2} Y+C X Y^{2}+D Y^{3}\right)\left(\alpha_{1} Q-\beta_{1} P\right)^{3}\left(\alpha_{2} Q-\beta_{2} P\right)^{3}\left(\alpha_{3} Q-\beta_{3} P\right)^{3}}{\left(\alpha_{1} \beta_{2}-\beta_{1} \alpha_{2}\right)^{2}\left(\alpha_{1} \beta_{3}-\beta_{1} \alpha_{3}\right)^{2}\left(\alpha_{2} \beta_{3}-\alpha_{3} \beta_{2}\right)^{2}},
$$

where the 4 points have projective coordinates $\left(\alpha_{1}: \beta_{1}\right),\left(\alpha_{2}: \beta_{2}\right),\left(\alpha_{3}\right.$ : $\left.\beta_{3}\right),(P: Q)$, the last being the coordinates of the distinguished element of the 4-tuple, and

$$
A X^{3}+\cdots+D Y^{3}=\left(\beta_{1} X-\alpha_{1} Y\right)\left(\beta_{2} X-\alpha_{2} Y\right)\left(\beta_{3} X-\alpha_{3} Y\right) .
$$

Note that replacing $\left(\alpha_{i}, \beta_{i}\right)$ by $\left(\lambda_{i} \alpha_{i}, \lambda_{i} \beta_{i}\right)$ leaves $Z$ unchanged, while replacing $(P, Q)$ by $(\lambda P, \lambda Q)$ changes $Z$ to $\lambda^{5} Z$. So the family of genus 1 curves lies in the $\mathbf{P}^{1}$ bundle over $X_{4}^{\prime}$ that is the pullback, by the projection $X_{4}^{\prime} \rightarrow \mathbf{P}^{1}$, of the rational normal scroll $\Sigma_{5} \rightarrow \mathbf{P}^{1}$, which is obtained from the degree 5 line bundle over $\mathbf{P}^{1}$ by completing each fiber $\mathbf{C}$ to a projective line.

More generally, if we consider the cover $X_{2 g+2}^{\prime}$ of $X_{2 g+2}$ corresponding to the subgroup $S_{2 g+1} \subset S_{2 g+2}$ stabilizing one point, the restriction $r^{*} y$ of the nontrivial element $y \in H^{1}\left(B\left(2 g+2, S^{2}\right), \mathbf{Z} / 2\right)$ becomes the reduction of a mod 4 class, so $\beta r^{*} y=0$. The family of curves can be constructed analogously to (1) in a $\mathbf{P}^{1}$ bundle over $X_{2 g+2}$ pulled back from the rational normal scroll $\Sigma_{4 g^{2}+g}$.

\section{ACKNOWLEDGMENT}

The question addressed arose during interesting discussions with $F$. R. Cohen and R. Hain. There is some overlap with [C1, C2, BCT]. I would like to thank O. Gabber who pointed out a blunder, and the I.H.E.S. and the I.A.S. for their hospitality and support.

\section{REFERENCES}

[A] V. Arnold, On some topological invariants of algebraic functions, Trans. Moscow Math. Soc. 21 (1970), 30-52.

[B] J. Birman, Braids, links and mapping class groups, Ann. of Math. Stud., vol. 82, Princeton Univ. Press, Princeton, NJ, 1975.

[BCT] C. Bödigheimer, F. R. Cohen, and L. R. Taylor, On the homology of configuration spaces, Topology 28 (1989), 111-123.

[Br] K. S. Brown, Cohomology of groups, Springer-Verlag, 1982.

[C1] F. R. Cohen, Applications of loopspaces to some problems in topology, preprint.

[C2] __ On the mapping class groups for a punctured sphere and a surface of genus 2, preprint.

Department of Mathematics, University of California, Los Angeles, California 90024 E-mail address: geoff@math.ucla.edu 